\title{
Recent advances in CZT strip detectors and coded mask imagers
}

\author{
J.L. Matteson ${ }^{1}$, D.E. Gruber ${ }^{1}$, W.A. Heindl ${ }^{1}$, M.R. Pelling ${ }^{1}$, L.E. Peterson ${ }^{1}$, R.E. Rothschild ${ }^{1}$, R.T. Skelton ${ }^{1}$, \\ P.L. Hink ${ }^{2}$, K.R. Slavis ${ }^{2}$, W.R. Binns ${ }^{2}$, T. Tumer ${ }^{3}$, and G. Visser ${ }^{3}$ \\ 1 University of California San Diego, La Jolla, CA 92093-0424, U.S.A. \\ e-mail: jmatteson@ucsd.edu * \\ 2 Washington University, St. Louis, MO 63130-4899, U.S.A. \\ 3 Nova R\&D, Inc., Riverside, CA 92507 and University of California Riverside, Riverside, CA 92521, U.S.A.
}

Received December 29, 1998; accepted May 18, 1999

\begin{abstract}
The UCSD, WU, UCR and Nova collaboration has made significant progress on the necessary techniques for coded mask imaging of gamma-ray bursts: position sensitive CZT detectors with good energy resolution, ASIC readout, coded mask imaging, and background properties at balloon altitudes. Results on coded mask imaging techniques appropriate for wide field imaging and localization of gamma-ray bursts are presented, including a shadowgram and deconvolved image taken with a prototype detector/ASIC and MURA mask.
\end{abstract}

Key words: balloons — instrumentation: detectors — gamma-rays: bursts

\section{Position-sensitive CZT detector}

Recent advances in CdZnTe (CZT) detector technology make gamma-ray burst localization possible using a coded mask imager consisting of a position sensitive CZT detector plane in conjunction with a coded mask. A prototype position-sensitive CZT detector (Fig. 1) that uses crossed-strip readout with 22 electrodes on each face has been developed (Matteson et al. 1998a). The strip pitch of $500 \mu \mathrm{m}$ yields $0.5 \mathrm{~mm} \times 0.5 \mathrm{~mm}$ pixels. Each anode and cathode strip is separately read out by its own preamplifier and electronic chain, contained in one of two 32 channel ASICs from Nova R\&D, Inc, called RENAs (Readout Electronics for Nuclear Applications), or in discrete electronics. Between the anode strips is a set of "steering electrodes", which are biased negative relative to the anodes to produce a well-defined electric field in the gap between the anodes, which improves charge collection.

\section{Energy resolution}

A set of X-ray and gamma-ray lines from 14 to $184 \mathrm{keV}$ were measured by the anodes of the prototype detector (Fig. 2). The lines are Gaussian-like at all energies. Energy

Send offprint requests to: J.L. Matteson

* This research was supported by NASA Grants NAG5-5111, NAG5-5114, and NGT5-50170.

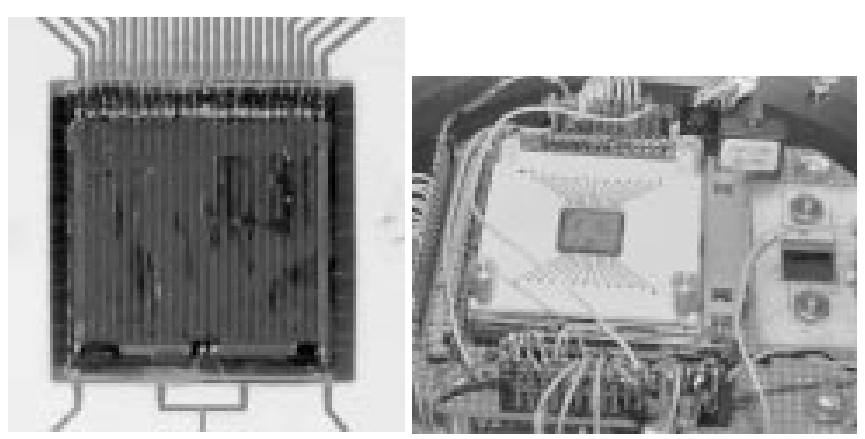

Fig. 1. Prototype detector $\left(12 \times 12 \times 2 \mathrm{~mm}^{3}\right)$. Left: Anode side; steering electrodes can be seen between anode strips. Right: Detector installed in platform used in laboratory and balloon flight tests. Strip detector left of center (cathode side on top) and planar detector at far right

resolution is $\sim 3 \mathrm{keV}$ at low energies, increasing to $\sim 6 \mathrm{keV}$ at high energies. These profiles are in marked contrast to those typically obtained in planar CZT detectors above $\sim 60 \mathrm{keV}$, where incomplete charge collection due to hole trapping results in tailing and poor spectral performance. The prototype detector's performance is similar to "spectrometer grade" CZT, which is expensive and only available in small sizes, $\sim 1 \mathrm{~cm}$. However, it is made of inexpensive "discriminator grade" CZT, which is available in large sizes, e.g., $32 \mathrm{~mm}$. Our advanced techniques produce spectrometer grade performance from discriminator grade material.

\section{Coded mask imaging}

A coded mask imager was developed for laboratory tests. Here the prototype detector with the RENA readout, a prototype coded mask $(23 \times 23$ MURA), and a small source of $41 \mathrm{keV}$ X-rays, $0.75 \mathrm{~mm}$ diameter ${ }^{153} \mathrm{Gd}$, were mounted in an optical bench, and their geometry was adjusted so the divergent X-ray beam magnified the mask's unit cells to 1300 microns at the detector. Since the shadowgram size is $60 \times 60$ pixels and the detector is $22 \times 22$ pixels, the mask/source combination was moved to several positions relative to the detector to record the full shadow- 


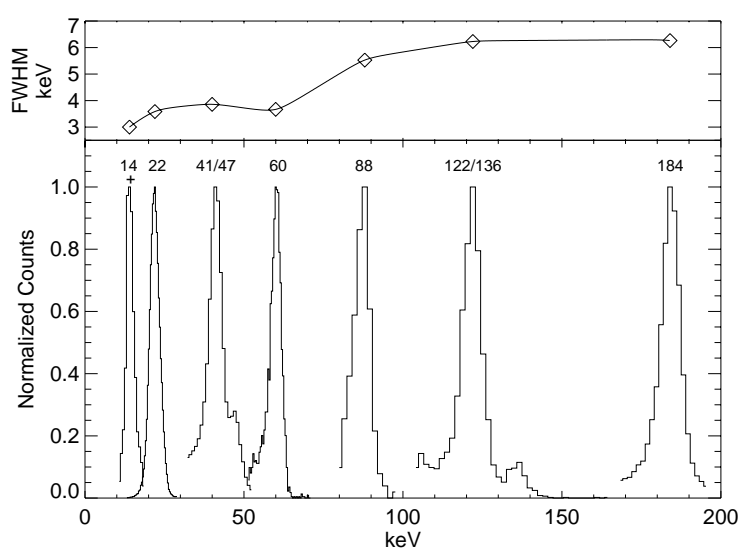

Fig. 2. Line profiles and FWHM energy resolution at several energies. All were measured with discrete laboratory electronics (Amptek A-250 preamps) except the $\mathbf{1 4} \mathbf{~ k e V}$ line which was measured with the RENA-type ASIC

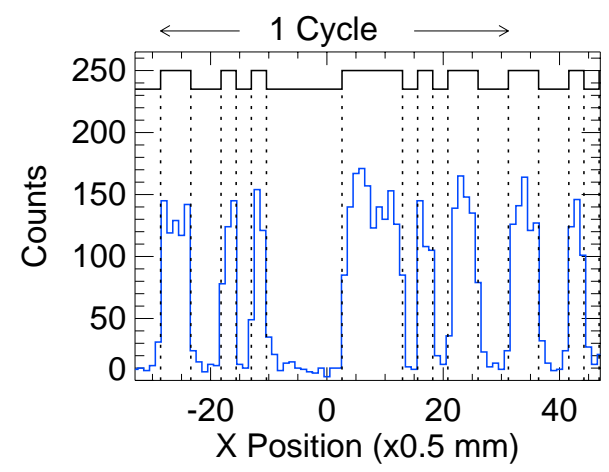

Fig. 3. A 1-dimensional cut of the shadow of the mask along one cathode strip. $X$-position corresponds to anode strip number. The mask pattern is indicated at the top, making clear the precise position resolution of the detector. Background is present at the level of $\sim 10 \mathrm{cts}$

gram. The shadow of the mask was cast upon the detector, and the counts versus position along one strip is shown in Fig. 3. The transitions from blocked to unblocked mask elements are at precisely the correct positions, showing the detector's high spatial accuracy, and the modulation transfer is $100 \%$. Unblocked counts are consistent with a constant value, which demonstrates the uniformity of response across the detector.

Shadowgrams of the mask were measured by the detector and then deconvolved to obtain a correlation image. Figure 4 shows the resulting shadowgram and image. All the mask features were accurately recorded by the shadowgram. Systematic noise in the image is undetectably small and limited to $<0.5 \%$ of the source peak. Thus, systematic errors can be expected to be minimal in the reconstruction and analysis of images. See Matteson et al. (1998b) for further information on imaging results.
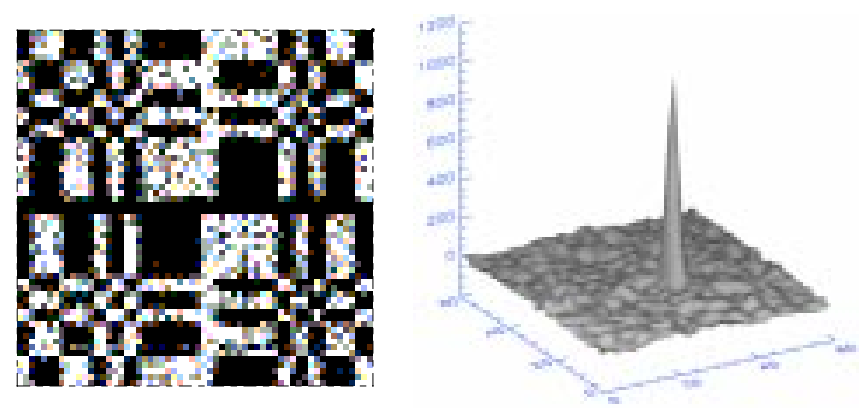

Fig. 4. Left: $60 \times 60$ pixel shadowgram of one coded mask cycle measured by the prototype coded mask imager with $41 \mathrm{keV}$ $\mathrm{X}$-rays. One mask cell equals 2.6 pixels. High counts are indicated by white. Bright regions have $\sim 15 \mathrm{cts} /$ pixel, dark regions $\sim 0.7 \mathrm{ct} /$ pixel. Right: Deconvolved image from shadowgram. $X$ and $Y$ units correspond to detector pixels

\section{Gamma-ray burst localization studies}

Rapid and accurate localization of bursts is a very important requirement for a wide field coded mask imager, and these capabilities were studied in detailed model calculations for cases near the detection threshold. These simulations addressed burst detection, identification, and localization for an imager with a $60^{\circ} \times 60^{\circ} \mathrm{FCFOV}, 129 \times 127$ URA, $369 \mathrm{~cm}^{2}$ detector, and $150 \mathrm{c} / \mathrm{s}$ background from $50-200 \mathrm{keV}$ (Slavis et al. 1998, give results on balloon flight background tests.) Two variables were considered: the ratio of burst count rate to background rate, and integration time. The three ratio values of $1: 2,2: 1$, and 10:1 were used and the integration time was inferred from the number of background counts.

The results show that for all ratios, when the expected detection significance is $5 \sigma$, the fraction of bursts correctly localized is $>80 \%$ and the RMS position uncertainty is $4.3^{\prime}$. Some of these cases have as few as 70 counts in the burst. One thousand burst counts, and negligible background, corresponds to a $22 \sigma$ detection and $\sim 1^{\prime}$ localization accuracy, and ten thousand counts yields an accuracy of $\sim 0.4^{\prime}$.

\section{References}

Matteson J.L., Duttweiler F., Huszar G.L., et al., 1998a, in: Hard X-ray and Gamma-Ray Detector Physics and Applications, Doty F.P. and Hoover R.B. (eds.), Proc. SPIE 3446, 192

Matteson J.L., Gruber D.E., Heindl W.A., et al., 1998b, in: EUV, X-ray, and Gamma-ray Instrumentation for Astronomy IX, Siegmund O.H.W. and Gummin M.A. (eds.), Proc. SPIE 3445, 445

Slavis K.R., Dowkontt P.F., Duttweiler F., et al., 1998, in: EUV, X-ray, and Gamma-Ray Instrumentation for Astronomy IX, O.H.W. Siegmund and M.A. Gummin (eds.), Proc. SPIE 3445, 169 